\title{
Factors that influence the duration of symptom resolution in COVID-19 patients in Ethiopia: A follow up study involving 60 symptomatic cases.
}

Saro Abdella Abrahim ( $\sim$ saroabrahim@gmail.com )

Ethiopian Public Health Institute https://orcid.org/0000-0001-7167-4043

Masresha Tessema

Ethiopian Public Health Institute

Eshetu Ejeta

Ethiopian Public Health Institute

Mahammed Ahmed

Ethiopian Public Health Institute

Atkure Defar

Ethiopian Public Health Institute

Alemayehu Hussen

Ethiopian Public Health Institute

Getachew Demoz

Eka Kotebe General Hospital, COVID-19 Isolation and Treatment Center

\section{Eskinder Degu}

Ethiopian Public Health Institute

Mulugeta Aseratie

Ethiopian Public Health Institute

\section{Belay Merga}

Ethiopian Public Health Institute

\section{Enatenesh Dillnessa}

Ethiopian Public Health Institute

\section{Susan Whiting}

College of Pharmacy and Nutrition, University of Saskatchewan, Saskatoon

\section{Tegene Regassa}

Ethiopian Ministry of Health

Derege Duguma

Ethiopian Ministry of Health 
Keywords: Sign, Symptom, Resolution, Recovery

Posted Date: December 31st, 2020

DOI: https://doi.org/10.21203/rs.3.rs-138076/v1

License: (c) (1) This work is licensed under a Creative Commons Attribution 4.0 International License. Read Full License 


\section{Abstract}

Background: The newly identified virus, Severe Acute Respiratory Syndrome Corona Virus-two (SARS-CoV2) has claimed more than a million lives worldwide since it was first recognized in Wuhan, China in December 2019. Understanding the clinical features of COVID-19 and duration for resolution of symptoms is crucial for isolation of patients and tailoring public health messaging, interventions, and policy. Therefore, this study aims to assess the median duration of COVID-19 signs and symptoms resolution and explore it's predictors among symptomatic COVID-19 patients in Ethiopia

Methods: A hospital-based prospective cohort study involving 60 COVID-19 cases was conducted at Eka Kotebe General Hospital, COVID-19 Isolation and Treatment Center. The study participants were all symptomatic COVID-19 adult patients admitted to the hospital from March 18 to June 27, 2020. Physicians at the center recorded the data using a log sheet. Cox proportional-hazards regression model was conducted. Statistical significance was defined at $P<0.05$. All analyses were done using STATA version 16.1 software.

Results: A total of 60 symptomatic COVID-19 patients with a mean age of 34.8 years $( \pm 1.8)$ were involved in the study. The median duration of symptom resolution of COVID-19 was seven days with a minimum of two and a maximum of 68 days. Sex and Body Mass Index (BMI) were statistically significant predictors of the symptom resolution. The hazard of having delayed sign or symptom resolution in males was $55 \%$ higher than in females $(P=0.039, \mathrm{Cl}: 0.22$ to 0.96$)$ and the hazard of the delayed sign or symptom in those with $\mathrm{BMI} \geq 25$ was $48 \%$ higher than in those with $\mathrm{BMI}<25$ ( $\mathrm{P}=0.051 ; \mathrm{Cl}: 0.272$ to 1.003). In this study, age and comorbidity had no association with the duration of sign or symptom resolution in COVID-19 patients.

Conclusions: The median duration of COVID-19 symptom resolution was seven days. Being male or having a $\mathrm{BMI} \geq 25$ were predictors for having a delayed sign or symptom resolution time. Therefore, understanding the duration of COVID-19 sign or symptom resolution helps to guide the patient isolation period and prioritize COVID-19 patients to be shielded.

\section{Background}

The newly identified virus, Severe Acute Respiratory Syndrome Corona Virus-two ( SARS-CoV-2) has claimed more than 700,000 lives worldwide since it was first recognized in Wuhan, China in December 2019[1]. Currently, there is no vaccine or cure that has passed all clinical trials required for approval. Countries all over the world have put in place preventive public health strategies to mitigate the devastating impact of the disease on their health system[2].

Transmission of the virus occurs by close contact through respiratory droplets, by direct contact with infected persons, or by contact with contaminated objects and surfaces[3]. Isolation of COVID-19 cases is a control measure in many countries limiting the spread of the virus.

According to the Centers for Disease Control and Prevention (CDC) recommendation, patients are 
discharged from isolation centers based on two consecutive negative reverse transcriptase polymerase chain reaction (rRT-PCR) test results of nasopharyngeal swabs obtained at least 24 hours apart, and resolution of respiratory symptoms and fever without the use of fever- reducing medicine[4].

On May 27, 2020, World Health Organization (WHO) put forward new criteria for discharging patients from isolation. The resolution of symptoms is taken as a criteria to discharge patients without laboratory tests. WHO recommends discharging symptomatic patients ten days after symptom onset, plus at least 3 days without symptoms (without fever and respiratory symptoms); and discharge of asymptomatic patients ten days after first positive test result without requiring further testing[5].

Understanding the clinical features of COVID-19 and duration for resolution of symptoms is crucial for isolation of patients and tailoring public health messaging, interventions, and policy. Based on the report of the WHO-China mission on Corona Virus Disease 2019, the top five typical signs and symptoms include fever, dry cough, fatigue, sputum production and shortness of breath[3].

In a study done among COVID-19 patients, the mean duration of COVID-19 symptoms of mild and moderate patients who eventually recovered was $11.5 \pm 5.7$ days [6]. WHO reported that the median duration for symptoms to resolve takes 2 weeks while it takes 3-6 weeks for patients with severe or critical disease[3]. However, a study conducted among outpatients with COVID-19 in a Multistate Health Care Systems Network in the United States reported that mild cases took at least 2 weeks for everyone to return to their baseline health. The duration of the symptoms resolution was affected by factors such as obesity, reporting of three or more chronic illnesses, and psychiatric illnesses [7]. In terms of factors associated with severe progression of COVID-19, older age, male sex, presence of underlying diseases were reported in several studies[6, 8, 9].

There are various studies that illustrated the duration of viral shedding among COVID-19 Patients. According to these studies, the median duration of viral shedding ranged from 8 to 30 days while the longest duration ranged from 37 to $47[10-17]$.

The duration of viral shedding is a potential indicator on the infectivity and transmissibility of epidemic diseases. It is also one of the significant criteria for discharge and provides vital information for effective infection prevention and control $[9,18]$. According to studies from Beijing and Wuhan, the median duration of recovery from the virus after symptoms resolution is 2.5 days and 8 days $[19,20]$. This indicates that patients continued to be virus positive even after the resolution of symptoms

Some of the studies conducted in China and western countries, revealed that older age, delayed initiation of antiviral treatment, highest temperature at admission, male sex, CHD co-morbidity, decreased albumin levels were among important factors that affect the duration of shedding of the virus[11, 14, 16, 21]. Corticosteroid treatment has also been indicated as predictors of longer duration of clearing of the virus from the body[12], however, another study claimed that low-to-moderate dosage of corticosteroid had little effect on the duration of viral excretion[11]. 
This study aims to explore predictors of symptom resolution among symptomatic COVID-19 patients in Ethiopia for the purpose of case management and prevention measures. The findings can be used to tailor effective public health messages and adopt strategies for treatment and prevention measures.

\section{Methods}

\section{Study Design and settings}

A hospital-based prospective cohort study involving 60 COVID-19 cases was conducted at Eka Kotebe General Hospital, COVID-19 Isolation and Treatment Center. The Center is the first hospital designated to manage positive COVID-19 cases in Ethiopia. It has the capacity of admitting 600 cases. During the study period, all individuals tested positive for SARS-CoV-2 on real-time reverse-transcriptase-polymerase chain reaction (rRT-PCR) were used to be admitted to the hospital regardless of sign and symptom development.

In this study, the dependent variable was duration of symptom resolution whereas independent variables were age, sex, comorbidities, and body mass index.

\section{Study participants}

The study participants were all symptomatic COVID-19 adult patients admitted to the Eka Kotebe General Hospital, COVID-19 Isolation and Treatment Center from March 18 to June 27, 2020. All COVID-19 cases who manifested/reported any illness were invited to participate in the study. Recovered symptomatic participants in the center during the study period were considered for analysis, data of those deceased were excluded.

\section{Sampling and study period}

All symptomatic COVID-19 cases who were admitted to the Eka Kotebe General Hospital, COVID-19 Isolation and Treatment Center during the study period and who gave consent to participate in the study were included. The study enrolled cases and followed up from March to June 2020.

The symptomatic case was defined as any SARS-CoV-2 positive person diagnosed by rRT-PCR with at least one sign or symptom for COVID-19. The signs and symptoms included but not limited to cough, fever, headache, muscle pain, and shortness of breath.

\section{Data collection}

A log sheet to record patients' symptom status was prepared. Physicians at the Eka Kotebe General Hospital, COVID-19 Isolation and Treatment Center recorded the COVID-19 signs and symptoms status of 
all patients every day. On-site data entry was conducted using tablets by trained data collectors in the facility, and data were transferred to the Ethiopian Public Health Institute Server through the REDCap system.

\section{Data management and analysis}

Continuous variables were expressed as mean \pm standard deviation (SD) for the normally distributed data or median with inter-quartile (IQR) for the skewed data. Descriptive analysis of survival data was presented graphically using Kaplan-Meier (KM) estimator. Log-Rank test was used to compare the survival experience of different categories of covariates. The proportional hazard assumption (PHA) was checked using Schoenfeld residual test. Cox proportional-hazards regression model was conducted to determine the potential risk factors associated with the time to sign and symptom resolution among symptomatic COVID-19 cases. Statistical significance was defined as $P<0.05$. All analyses were done using STATA version 16.1 software.

\section{Ethical clearance}

The study protocol was developed by the study team and reviewed by the Ethiopian Public Health Institute's Institutional Review Board. The protocol was approved (Ethics Ref. No. EPHI 6.13/690). Informed consent from every participating case was obtained. Data security, participants' confidentiality were maintained at all levels of data management.

\section{Results}

A total of 60 symptomatic COVID-19 patients with a mean age of 34.8 years $( \pm 1.8)$ were followed up to see symptom or sign resolution date. The majority $(80 \%)$ of participants were male. The higher proportion of the participants $(67 \%)$ had BMl 25 , and $20 \%$ of them had comorbidity (Table 1 ).

Table 1 Background information of the study participants 


\begin{tabular}{llll}
\hline Variables & Category & Frequency (n) & Percent (\%) \\
\hline Age & Mean $=34.8 \pm 1.8$ & & \\
Sex & Female & 12 & 20.0 \\
& Male & 48 & 80.0 \\
BMI & $<25$ & 39 & 67.2 \\
& $\geq 25$ & 19 & 32.8 \\
Comorbidities & Have at least one comorbidity & 12 & 20.0 \\
& Have no comorbidity & 48 & 80.0 \\
\hline
\end{tabular}

Out of the 12 participants who had co-morbidities, five were self-reported Cancer patients, three HIV/AIDS and another three were hypertensive patients (Table 2).

Table 2 Comorbidities Reported and Median Duration of Symptom Resolution

\begin{tabular}{lccc} 
Comorbidity & Frequency & Median days of symptom resolution & SD \\
& 5 & 7 & \\
\hline Cancer & 3 & 15 & 3.29 \\
HIV/AIDS & 3 & 24 & 8.16 \\
Hypertension & 1 & 5 & 2.45 \\
Heart Disease & 1 & 6 & 0 \\
Asthma & 1 & 6 & 0 \\
Chronic Liver Disease & & & 0 \\
\hline
\end{tabular}

Respiratory and neurological illnesses were among the most commonly reported signs and symptoms in the study participants. The majority of the participants (40\%) had cough and only a few had reported gastrointestinal illness (8.3\%). (Figure 1)

Figure 1: Percentage of signs and symptoms in the study participants

All the study participants had sign or symptom before the date of diagnosis for SARS-CoV-2 by rRT-PCR. The median duration between the date of diagnosis and date of symptom onset was 3 days, ranging from 1-25 days. The majority of the participants (88\%) were diagnosed with the virus in nine days after the onset of sign or symptom; $66 \%$ in five days and $22 \%$ in $5-9$ days. Few $(n=3,5 \%)$ were diagnosed after 20 days of symptom onset (Figure 2). 
Figure 2: Duration from sign or symptom onset to diagnosis

The median duration of sign or symptom resolution of COVID-19 in the study participants was seven days with a minimum of two and a maximum of 68 days. The median duration of symptom resolution in males and females was similar ( 7 days for males and 6 days for females). Participants with BMI>25 took a longer duration to get free of their respective signs or symptoms compared with those with $\mathrm{BMI}<25$. Individuals with at least one comorbidity showed less duration for resolution of signs or symptoms (Table 3).

Table 3: Median Duration of Symptom Resolution

\begin{tabular}{|c|c|c|c|}
\hline Variables & Category & $\begin{array}{l}\text { Median duration of } \\
\text { symptom resolution* }\end{array}$ & $\begin{array}{l}\text { Log-rank } \\
\text { test, } \mathrm{P} \text { - } \\
\text { value }\end{array}$ \\
\hline
\end{tabular}

Date from symptom onset Min=2,

7

to resolution $\operatorname{Max}=68$

Date from symptom $\quad$ Min=0, 16

resolution to $\mathrm{PCR}$ negative $\mathrm{Max}=77$

test

$\begin{array}{lllll}\text { Age } & \text { Mean }= & & \\ & 34.8 \pm 14.2 & & \\ \text { Sex } & \text { Female } & 6 & 0.4130 & {[3,17]} \\ & \text { Male } & 7 & & {[6,8]} \\ \text { BMI } & <25 & 7 & 0.0481 & {[6,7]} \\ & \geq 25 & 10 & 0.2596 & {[4,24]} \\ \text { Co-morbidities } & \text { Have at least } & 10 & & {[6,17]} \\ & \text { one } & & \\ & \text { comorbidity } & & {[6,8]} \\ & \text { Have no } & & \end{array}$

* Symptom resolution duration was defined as duration from the first date of first sign or symptom onset to the last date of any sign or symptom. 
The median duration of identification of COVID-19 patients in community screening was three days after being symptomatic. The median duration of the symptoms resolution was seven days. The median duration of recovery from the virus, rRT-PCR negative test for SARS-CoV-2, was 16 days after symptom resolution (Figure 3).

Figure 3 Median Durations of Symptoms and Recovery

Controlling for influencing covariates, sex was found to be a significant predictor of sign or symptom resolution. The hazard of having delayed sign or symptom resolution in males was $55 \%$ higher than in females ( $P=0.039, \mathrm{Cl}: 0.22$ to 0.96$)$ and the hazard of the delayed sign or symptom in those with $\mathrm{BMI} \geq 25$ was $48 \%$ higher than in those with $\mathrm{BMI}<25(\mathrm{P}=0.051 ; \mathrm{Cl}$ : 0.272 to 1.003$)$. $\mathrm{BMI}$ was marginally significant predictor of the symptom resolution. Age and comorbidity had no association with a delayed sign or symptom in COVID-19 patients (Table 4).

Table 4: Predictors of duration of symptom resolution among COVID-19 patients: Cox Proportional Hazards Analysis ( $\mathrm{n}=58)$

\begin{tabular}{|c|c|c|c|}
\hline Variables & Categories & AHR(95\%CI) & p-valu \\
\hline Age & $34.8 \pm 14.2$ & $0.99(0.97,1.01)$ & 0.611 \\
\hline \multicolumn{4}{|l|}{ Sex } \\
\hline & Male & $(0.22,0.96)$ & 0.039 \\
\hline & Female & 1 & \\
\hline
\end{tabular}

Comorbidity

No comorbidity $\quad 1$

At least one comorbidity $\quad 0.69(0.33,1.44) \quad 0.32$

\begin{tabular}{llll} 
BMI & $<25$ & 1 & \\
& $\geq 25$ & $0.52(0.27,1.003)$ & 0.051 \\
\hline
\end{tabular}

\section{Discussion}

In this study, we have determined the median duration of symptoms resolution of COVID-19 patients and explored predictors of symptom resolution among symptomatic COVID-19 patients in Ethiopia. The median duration of sign or symptom resolution of COVID-19 in the study participants was 7 days with a minimum of two and a maximum of 68 days. Controlling for influencing covariates, sex was found to be 
a significant predictor of sign or symptom resolution. BMI was marginally significant predictor of the symptom resolution. In this study, age and comorbidity had no association with a delayed sign or symptom in COVID-19 patients.

According to Chang et al[19] and Siordia Jr et al [22], the commonly reported symptoms in patients with COVID-19 were fever, cough, and dyspnea. Headache, loss of smell, and nasal obstruction were the most common clinical presentations of mild to moderate patients in Europe [6]. While fever, cough, dyspne and fatigue were the most common clinical presentation in Asian [23, 24].Our study also reported a similar finding in which cough, fever, headache, and sore throat were among the most common signs and symptoms. Furthermore, this study finding showed that cancer, HIV/AIDS, and hypertension were the most common co-morbidities among study participants. Hypertension, diabetes mellitus, and cardiovascular disease were the most commonly reported co-morbidity according to a study done on the clinical feature of COVID-19 [22].

The median duration of symptom resolution in our study is comparable to a study done in Beijing, China by Chang et al that reported a median duration of eight days (IRQ 6.25-11.5) [19]. However, this study's median duration is lower than the median duration of a study done in Soonchunhyang University Seoul Hospital ( 10 days with a minimum of 2 to a maximum of 38 days[25], in European hospitals (11.5 with a standard deviation of 5.7 days)[6], and in the hospitals outside Wuhan(11 days) [26]. Socioeconomic status, patient's disease status, and sample size might be the possible reason for the difference.

Little information on predictors of duration of COVID-19 sign or symptoms resolution is yet available, the finding of this study revealed that sex and BMI were associated with the duration of COVID-19 symptoms resolution. The hazard of having delayed sign or symptom resolution in males was more likely than females. The reason might be that females are less likely to have complications related to viral infection because of innate immunity, steroid hormones, and sex hormones [6]. However, a study conducted on symptom duration and a risk factor for delayed return to usual health among outpatient with COVID-19 in the USA reported that there was no significant association between sex and return to usual health[7].

Our study also revealed that those COVID-19 patients with body mass index (BMI) $\geq 25$ have delayed duration of COVID-19 related sign or symptom resolution. This finding is similar to the study done in the USA and reported that those with a higher BMI were twice less likely to return to usual health state as compared with those with lower BMI $(<30)$ [7]. A retrospective study conducted on COVID-19 patients in New York City had also revealed that obese $(\mathrm{BMI} \geq 30)$ patients had a significantly higher rate of intensive care unit admission or death[27]. Moreover, a dose-response meta-analysis study conducted on body mass index and outcome in patients with COVID-19 indicated that BMI $\geq 30$ was associated with mortality and severity in patients with COVID-19[28].

This study showed that age was not significantly associated with the duration of COVID-19 sign or symptom resolution. However, a study conducted in the USA showed that older age was associated with delayed return to their usual health state [7]. The age-dependent defects in T-cell and B-cell function and excess production of type 2 cytokines leads to a deficiency in control of viral replication and more 
prolonged pro-inflammatory responses, which in turn lead to poor outcome [9, 29]. A prospective cohort study conducted on critically ill patients with laboratory-confirmed COVID-19 patients in New York City also reported that older age was more likely to develop progressive COVID-19 infection and to die [30]. In our study, the average age of participants was 34 years with a standard deviation of 1.8 and the age of $95 \%$ of the participants were less than 65 years. This could mask the possible association between age and symptom resolution.

\section{Limitations Of The Study}

Although using prospective follow up study is the strength of this study, the results shall be interpreted with caution. There could be a chance that duration of mild symptoms might be overlooked by individuals and not reported. Small sample size is also a limitation of this study.

\section{Conclusion}

Our findings showed that the median duration of COVID-19 sign or symptom resolution was seven days. Being male and having a $\mathrm{BMI} \geq 25$ have delayed sign or symptom resolution period. An average of two weeks were required to be cured of SARS-CoV-2 for symptomatic patients after symptom resolution in Ethiopia. Therefore, understanding the duration of COVID-19 sign or symptoms resolution helps to guide the patient isolation period and overall management of symptomatic COVID-19 patients.

\section{List Of Abbreviations}

BMI Body Mass Index

rRT-PCR Real-time reverse-transcriptase-polymerase chain reaction

SARS-COV-2 Sever Acute Respiratory Syndrome Corona Virus-two

WHO World Health Organization

\section{Declarations}

\section{Ethics approval and consent to participate}

The study protocol was developed by the study team and reviewed by the Ethiopian Public Health Institute's Institutional Review Board. The protocol was approved (Ethics Ref. No. EPHI 6.13/690).

All participants had provided informed consent to participate in the study. The lead authors affirms that the manuscript is an honest, accurate, and transparent account of the study being reported; that no 
important aspects of the study have been omitted; and that any discrepancies from the study as planned have been explained.

Availability of data and materials: The dataset used and analysed during this study will be made available from the corresponding author on reasonable request.

Competing interests: We declare that they have no competing interests

Funding: The authors did not receive any specific funding for this study.

Authors' contribution: SA and MT designed the study, SA, MT, EE, AD and AH performed the statistical analysis and wrote the first draft of the manuscript. All other co-authors contributed further to the development of the manuscript to the scientific standards. All authors read and approved for correspondence to publication.

Acknowledgment: We would like to thank staff at Eka Kotebe General Hospital, COVID-19 Isolation and Treatment Center for their valuable support during the study period.

\section{References}

1. WHO: Clinical management of COVID-19: interim guidance. In.: World Health Organization; 2020.

2. Güner R, Hasanoğlu I: COVID-19: Prevention and control measures in community. 2020, 50(Si-1):571577.

3. WHO: Report of the WHO-China Joint Mission on Coronavirus Disease 2019 (COVID-19) In.: World Health Organization; 2020.

4. Discontinuation of Transmission-Based Precautions and Disposition of Patients with COVID-19 in Healthcare Settings (Interim Guidance)

5. WHO: Criteria for releasing COVID-19 patients from isolation: Scientific brief. In.: World Health Organization; 2020.

6. Lechien JR, Chiesa-Estomba CM, Place S, Van Laethem Y, Cabaraux P, Mat Q, Huet K, Plzak J, Horoi $M$, Hans S et al: Clinical and epidemiological characteristics of 1420 European patients with mild-tomoderate coronavirus disease 2019. J Intern Med 2020, 288(3):335-344.

7. Tenforde MW, Kim SS, Lindsell CJ, Billig Rose E, Shapiro NI, Files DC, Gibbs KW, Erickson HL, Steingrub JS, Smithline HA et al: Symptom Duration and Risk Factors for Delayed Return to Usual Health Among Outpatients with COVID-19 in a Multistate Health Care Systems Network - United States, March-June 2020. MMWR Morbidity and mortality weekly report 2020, 69(30):993-998.

8. Voinsky I, Baristaite G, Gurwitz D: Effects of age and sex on recovery from COVID-19: Analysis of 5,769 Israeli patients. Journal of Infection 2020.

9. Zhou F, Yu T, Du R, Fan G, Liu Y, Liu Z, Xiang J, Wang Y, Song B, Gu X et al: Clinical course and risk factors for mortality of adult inpatients with COVID-19 in Wuhan, China: a retrospective cohort study. 
Lancet (London, England) 2020, 395(10229):1054-1062.

10. Chen X, Zhu B, Hong W, Zeng J, He X, Chen J, Zheng H, Qiu S, Deng Y, Chan JC: Associations of Clinical Characteristics and Treatment Regimens with Viral RNA Shedding Duration in Patients with COVID-19. International Journal of Infectious Diseases 2020.

11. Shi D, Wu W, Wang Q, Xu K, Xie J, Wu J, Lv L, Sheng J, Guo J, Wang K et al: Clinical Characteristics and Factors Associated With Long-Term Viral Excretion in Patients With Severe Acute Respiratory Syndrome Coronavirus 2 Infection: a Single-Center 28-Day Study. The Journal of infectious diseases 2020, 222(6):910-918.

12. Li TZ, Cao ZH, Chen Y, Cai MT, Zhang LY, Xu H, Zhang JY, Ma CH, Liu Y, Gao LJ et al: Duration of SARS-CoV-2 RNA shedding and factors associated with prolonged viral shedding in patients with COVID-19. Journal of medical virology 2020.

13. Warabi Y, Tobisawa S, Kawazoe T, Murayama A, Norioka R, Morishima R, Inoue T, Shimizu T, Takahashi K: Effects of oral care on prolonged viral shedding in coronavirus disease 2019 (COVID19). Special care in dentistry : official publication of the American Association of Hospital Dentists, the Academy of Dentistry for the Handicapped, and the American Society for Geriatric Dentistry 2020.

14. Yan D, Liu XY, Zhu YN, Huang L, Dan BT, Zhang GJ, Gao YH: Factors associated with prolonged viral shedding and impact of lopinavir/ritonavir treatment in hospitalised non-critically ill patients with SARS-CoV-2 infection. The European respiratory journal 2020, 56(1).

15. Qi L, Yang Y, Jiang D, Tu C, Wan L, Chen X, Li Z: Factors associated with duration of viral shedding in adults with COVID-19 outside of Wuhan, China: A retrospective cohort study. International Journal of Infectious Diseases 2020.

16. Zheng X, Chen J, Deng L, Fang Z, Chen G, Ye D, Xia J, Hong Z: Risk factors for the COVID-19 severity and its correlation with viral shedding: a retrospective cohort study. 2020.

17. van Kampen JJ, van de Vijver DA, Fraaij PL, Haagmans BL, Lamers MM, Okba N, van den Akker JP, Endeman H, Gommers DA, Cornelissen JJ: Shedding of infectious virus in hospitalized patients with coronavirus disease-2019 (COVID-19): duration and key determinants. medRxiv : the preprint server for health sciences 2020.

18. Ryoo SM, Kim WY, Sohn CH, Seo DW, Oh BJ, Lee JH, Lee YS, Lim KS: Factors promoting the prolonged shedding of the pandemic (H1N1) 2009 influenza virus in patients treated with oseltamivir for 5 days. Influenza and other respiratory viruses 2013, 7(5):833-837.

19. Chang D, Mo G, Yuan X, Tao Y, Peng X, Wang FS, Xie L, Sharma L, Dela Cruz CS, Qin E: Time Kinetics of Viral Clearance and Resolution of Symptoms in Novel Coronavirus Infection. American journal of respiratory and critical care medicine 2020, 201(9):1150-1152.

20. Fu Y, Han P, Zhu R, Bai T, Yi J, Zhao X, Tao M, Quan R, Chen C, Zhang Y: Risk Factors for Viral RNA Shedding in COVID-19 Patients. European Respiratory Journal 2020.

21. Hu F, Yin G, Chen Y, Song J, Ye M, Liu J: Corticosteroid, oseltamivir and delayed admission are independent risk factors for prolonged viral shedding in patients with Coronavirus Disease 2019. 2020. 
22. Siordia JA, Jr.: Epidemiology and clinical features of COVID-19: A review of current literature. Journal of clinical virology : the official publication of the Pan American Society for Clinical Virology 2020, 127:104357.

23. Cao Y, Liu X, Xiong L, Cai K: Imaging and clinical features of patients with 2019 novel coronavirus SARS-CoV-2: A systematic review and meta-analysis. 2020.

24. Huang C, Wang Y, Li X, Ren L, Zhao J, Hu Y, Zhang L, Fan G, Xu J, Gu X et al: Clinical features of patients infected with 2019 novel coronavirus in Wuhan, China. Lancet (London, England) 2020, 395(10223):497-506.

25. Park SY, Yun SG, Shin JW, Lee BY, Son HJ, Lee S, Lee E, Kim TH: Persistent severe acute respiratory syndrome coronavirus 2 detection after resolution of coronavirus disease 2019-associated symptoms/signs. The Korean journal of internal medicine 2020, 35(4):793-796.

26. Xu K, Chen Y, Yuan J, Yi P, Ding C, Wu W, Li Y, Ni Q, Zou R, Li X: Factors associated with prolonged viral RNA shedding in patients with COVID-19. Clinical Infectious Diseases 2020.

27. Hajifathalian K, Kumar S, Newberry C, Shah S, Fortune B, Krisko T, Ortiz-Pujols S, Zhou XK, Dannenberg AJ, Kumar R: Obesity is associated with worse outcomes in COVID-19: Analysis of Early Data From New York City. Obesity 2020.

28. Pranata R, Lim MA, Yonas E, Vania R, Lukito AA, Siswanto BB, Meyer M: Body mass index and outcome in patients with COVID-19: A dose-response meta-analysis. Diabetes \& metabolism 2020.

29. Opal SM, Girard TD, Ely EW: The immunopathogenesis of sepsis in elderly patients. Clinical infectious diseases : an official publication of the Infectious Diseases Society of America 2005, 41 Suppl 7:S504-512.

30. Cummings MJ, Baldwin MR, Abrams D, Jacobson SD, Meyer BJ, Balough EM, Aaron JG, Claassen J, Rabbani LE, Hastie $\mathrm{J}$ et al: Epidemiology, clinical course, and outcomes of critically ill adults with COVID-19 in New York City: a prospective cohort study. Lancet (London, England) 2020, 395(10239):1763-1770.

\section{Figures}




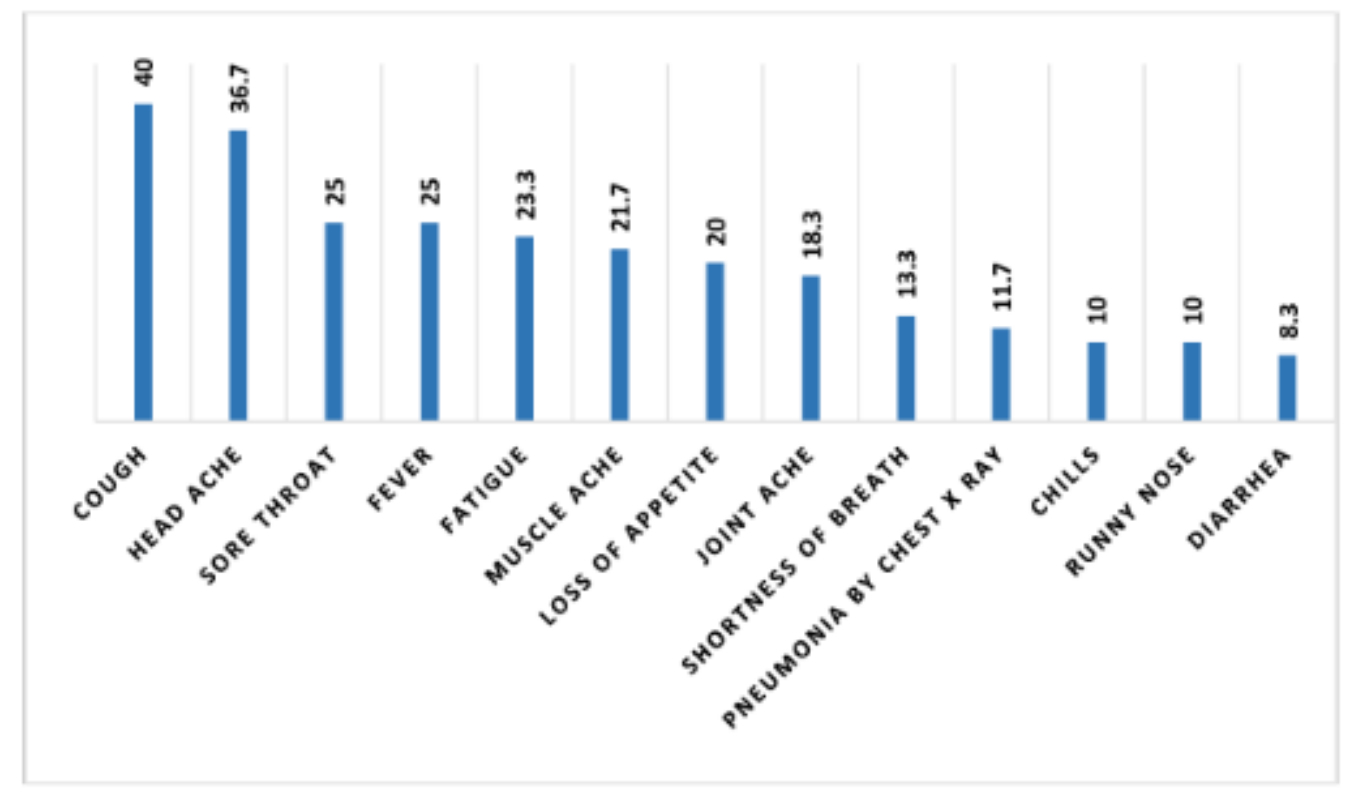

Figure 1

Percentage of signs and symptoms in the study participants

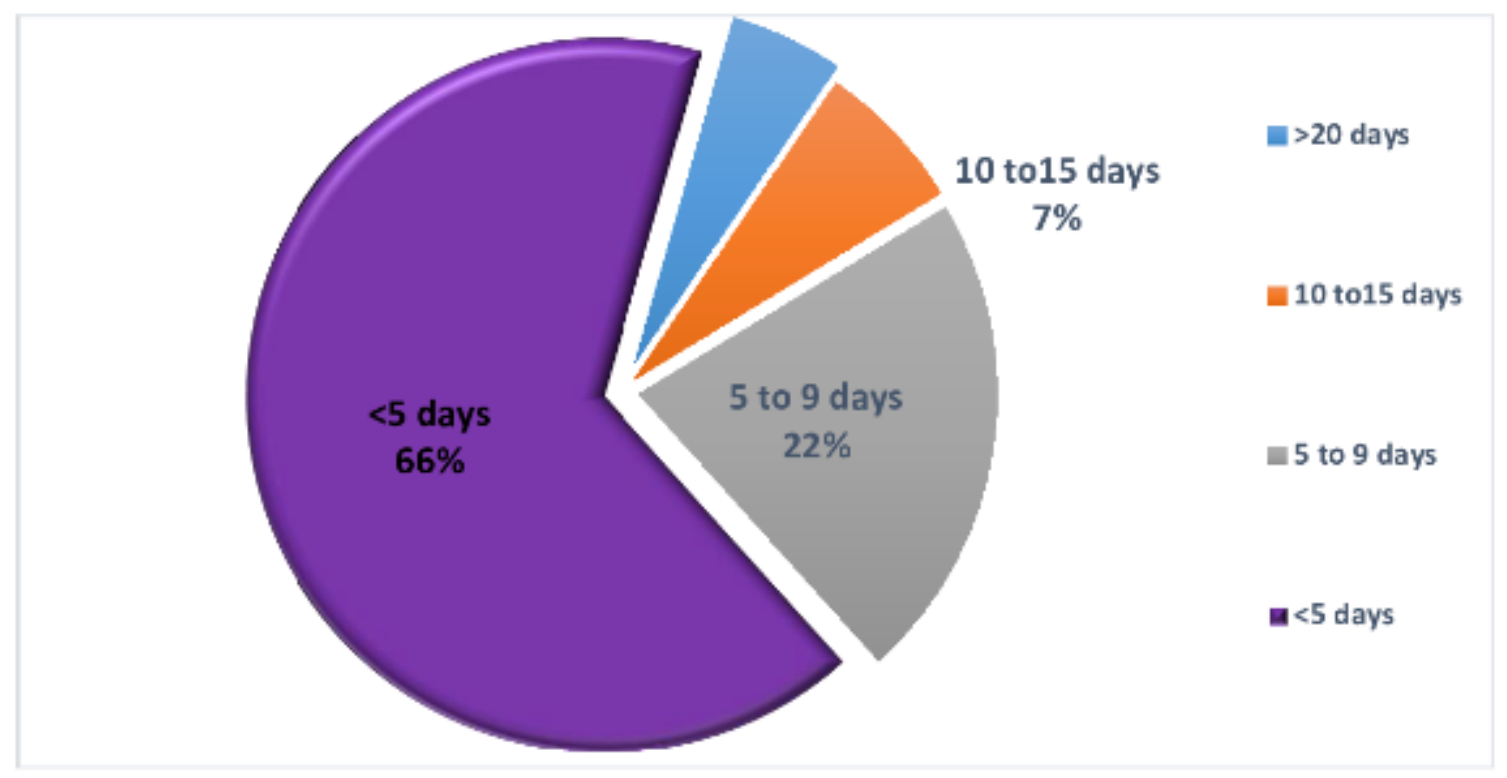

Figure 2

Duration from sign or symptom onset to diagnosis 


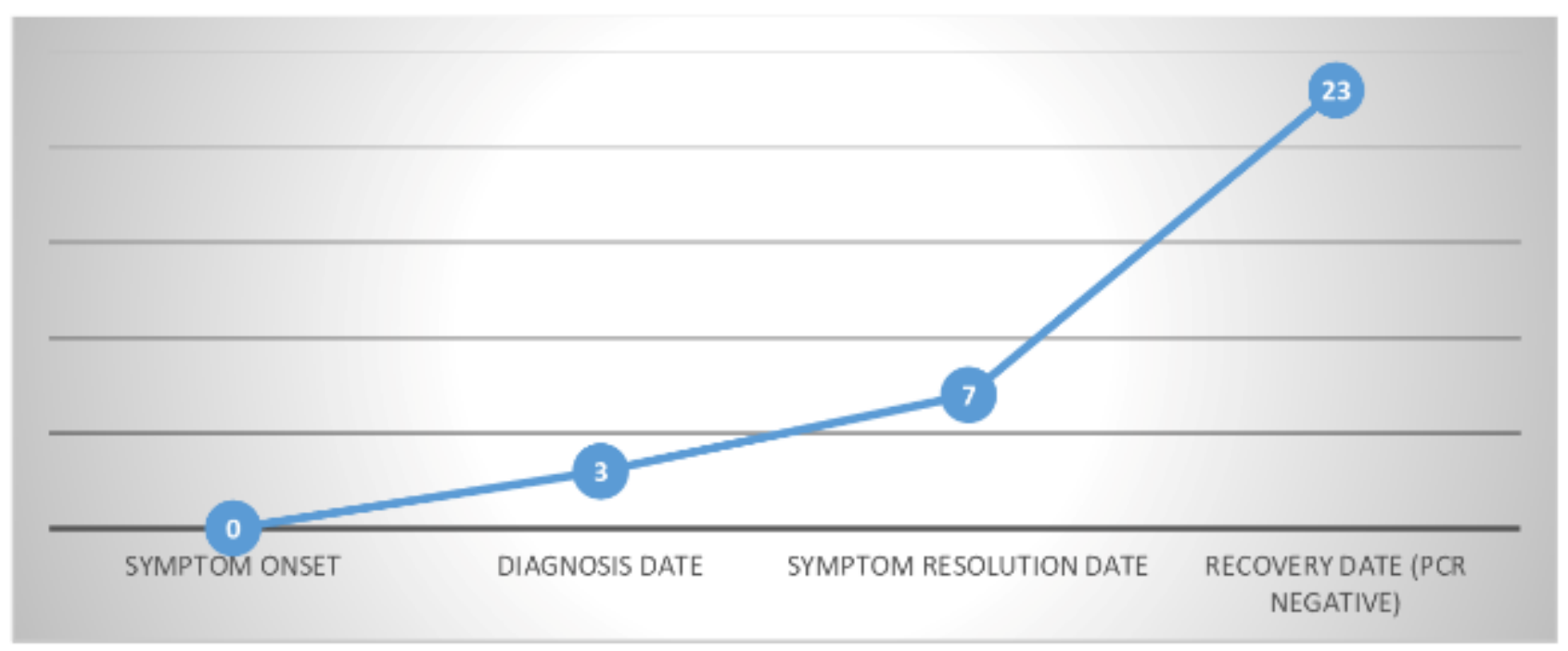

Figure 3

Median Durations of Symptoms and Recovery 